\title{
Normal Perfusion Pressure Breakthrough Following AVM Resection: A Case Report and Review of the Literature
}

\author{
T. E. O’Connor ${ }^{1}$, K. M. Fargen ${ }^{2}$, J. Mocco ${ }^{3}$ \\ ${ }^{1}$ College of Medicine, University of Florida, Gainesville, USA \\ ${ }^{2}$ Department of Neurological Surgery, University of Florida, Gainesville, USA \\ ${ }^{3}$ Department of Neurological Surgery, Vanderbilt University, Nashville, USA \\ Email: timocon@ufl.edu
}

Received August 22, 2013; revised September 22, 2013; accepted September 30, 2013

Copyright ( 2013 T. E. O’Connor et al. This is an open access article distributed under the Creative Commons Attribution License, which permits unrestricted use, distribution, and reproduction in any medium, provided the original work is properly cited.

\begin{abstract}
Objective: To report a patient's clinical course illustrative of the NPPB mechanism for hyperperfusion-induced injury. Methods: A 65-year-old female presented with a severe headache and was found to have a 6-cm right parietal AVM on imaging. The patient underwent staged, pre-operative embolization and the AVM was surgically resected without intra-operative complication. After the patient emerged from anesthesia she exhibited left hemiplegia and hemispatial neglect. Her systolic blood pressure (SBP) at that time was between $110-120 \mathrm{mmHg}$. SBP was reduced to 90 - 100 $\mathrm{mmHg}$ and the patient's symptoms resolved shortly thereafter. The patient's strict blood pressure goal was relaxed the next morning. However, with her SBP $110-120 \mathrm{mmHg}$ in the ensuing hours, the patient's left-sided neglect and hemiparesis returned. Her SBP was reduced again to $90-100 \mathrm{mmHg}$, leading to resolution of her symptoms. Results: This patient's clinical course supports the NPPB theory of hyperperfusion-induced injury. Despite CT imaging demonstrating no residual AVM following resection, the patient developed neurological deficits in the immediate postoperative period. Aggressive systemic hypotension improved clinical symptoms repeatedly, whereas a brief period of normotension triggered a return of neurological deficits. As a result, there was a direct correlation between fluctuations of neurological status and SBP. This case suggests that the intrinsic autoregulatory capacity was altered in our patient, and that aggressive hypotension was necessary to compensate for diminished autonomic reactivity. Conclusions: This case provides further evidence that NPPB plays a role in hyperperfusion-induced injury following AVM excision and that blood pressure control is vital in managing hyperemic complications following complete resection of cerebral AVMs.
\end{abstract}

Keywords: Blood Pressure; Hemorrhage; Intracranial Arteriovenous Malformations; Normal Perfusion Pressure Breakthrough

\section{Introduction}

Arteriovenous malformations (AVMs) are high-flow vascular shunts between arteries and veins that lack an intervening capillary bed. These lesions carry an inherent risk of spontaneous bleeding, with the natural incidence of hemorrhage reportedly 3\% - 4\% per year [1-3]. Despite advances in microsurgical techniques and perioperative care, surgical resection of cerebral AVMs carries an estimated risk of morbidity ranging from $12 \%$ to $20 \%$, and a risk of mortality ranging from $1 \%$ to $4 \%$ [4-7]. AVM obliteration normalizes the cerebral perfusion pressure of previously hypotensive vasculature, predisposing the surrounding environment to complications such as edema and hemorrhage [7-10].

There is currently an ongoing discussion regarding the underlying pathophysiologic mechanism behind cerebrovascular complications following AVM obliteration. Normal perfusion pressure breakthrough (NPPB) was initially proposed by Spetzler et al. in 1978 to explain the presence of hemodynamic alterations upon restoration of normal tissue perfusion following AVM ablation [8]. Spetzler et al. suggested that hypoperfusion could induce local vessels surrounding the nidus to chronically dilate and predispose the vascular territory to vasomotor paralysis. Upon restoration of normal perfusion following AVM resection, NPPB postulates that an impaired autoregulatory capacity may then be unable to compensate for increases in arterial flow and ultimately cause hyperemia, edema, or intracerebral hemorrhage [8].

Although empirical evidence supports the existence of autoregulatory dysfunction adjacent to the AVM nidus, 
further studies have demonstrated that complications often occur in the absence of vasomotor paralysis. Young et al. demonstrated that $\mathrm{CO}_{2}$ reactivity remained intact following AVM resection, and later determined cerebral blood flow could remain stable following pharmacologically induced increases in mean arterial pressure [11,12]. Debate remains on the significance of NPPB in the setting of hyperemic complications, and other mechanisms such as incomplete nidus obliteration [13], occlusive hyperemia [9,14], and increased capillary density [15,16] have been implicated as contributing factors.

We present a patient that developed left hemiplegia and hemispatial neglect after staged embolization and excision of an AVM that responded repeatedly to systemic hypotension. Postoperative imaging demonstrated cerebral edema without evidence of ischemic infarction, residual AVM or hemorrhage. This patient's clinical course supports the NPPB mechanism for hyperemic complications following AVM resection.

\section{Case Report}

A 65-year-old left handed female with chronic headaches presented with a severe right-sided headache that developed gradually over one week. She was neurologically intact on examination. MR imaging and digital subtraction angiography (DSA) demonstrated a 6-cm right parietal AVM classified as a Spetzler-Martin grade 3 (Spetzler and Martin 1986). Arterial feeders were most prominent from the right posterior cerebral artery but were also noted from the right anterior and middle cerebral arteries, as well as the superficial temporal artery (Figures 1 and 2). There was also involvement of the left anterior cerebral artery (ACA). Imaging demonstrated large, dilated venous drainage in the right parietal region that was primarily superficial without evidence of significant deep venous drainage.

The patient underwent staged, pre-operative embolization to reduce flow to the nidus. Uncomplicated transcatheter Onyx Liquid Embolic System (Covidien-ev3; Plymouth, MN) embolization of a medial posterior cerebral feeding artery was performed, followed one week later by Onyx embolization of feeders from the right ACA. After the second embolization, postoperative angiography demonstrated residual feeding vasculature off the middle cerebral artery in addition to persistent deep feeders from the PCA (Figures 3 and 4). The patient remained at her neurologic baseline following all embolizations.

The following day the AVM was surgically resected via a right interhemispheric approach without intra-operative event or complication. No fixed retractors were used during the procedure, and only gentle retraction was applied to the adjacent hemisphere to minimize the potential for retraction-induced injury. Intra-operative ex-

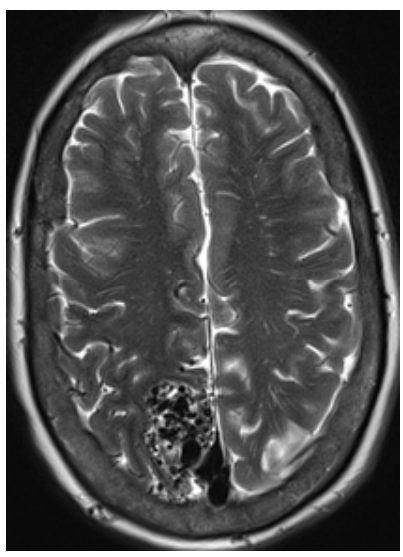

Figure 1. MRI of the brain at presentation demonstrating the right parietal AVM with large posterior draining veins.
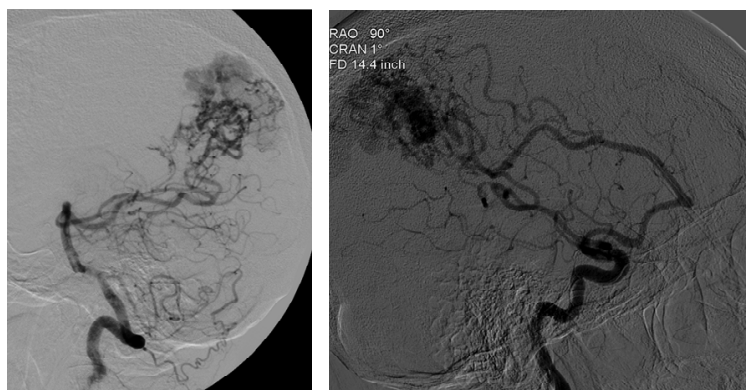

Figure 2. Lateral digital subtraction angiography after right vertebral artery injection (left panel) and right internal carotid artery injection (right panel) demonstrating prominent right PCA, MCA and ACA feeding vessels.

amination revealed no evidence of hemorrhage or brain injury. Intra-operative DSA demonstrated no evidence of residual AVM or abnormal residual arteriovenous shunting. These findings were consistent with complete AVM resection.

The patient was then emerged from anesthesia in standard fashion with an effort to maintain blood pressure at $120 \mathrm{mmHg}$ or below. On a delayed post-operative neurological examination, she exhibited $0 / 5$ strength in her left lower extremity with a left hemispatial neglect. Otherwise she was awake and oriented with full strength on her right side. Her systolic blood pressure at that time was between 110 and $120 \mathrm{mmHg}$. Emergent noncontrast CT imaging revealed pneumocephalus and bilateral hemisphere edema without evidence of acute hemorrhage (Figure 5). The patient was administered mannitol and hypertonic saline and initiated on dexamethasone. Systolic blood pressure was then reduced pharmacologically to 90 - $100 \mathrm{mmHg}$ with a labetalol infusion, and the patient's symptoms subsequently resolved shortly thereafter. Given that her neurologic examination had improved, the patient's strict blood pressure goal was relaxed the next morning and she was once again allowed to have systolic blood pressures at or below $120 \mathrm{mmHg}$. 


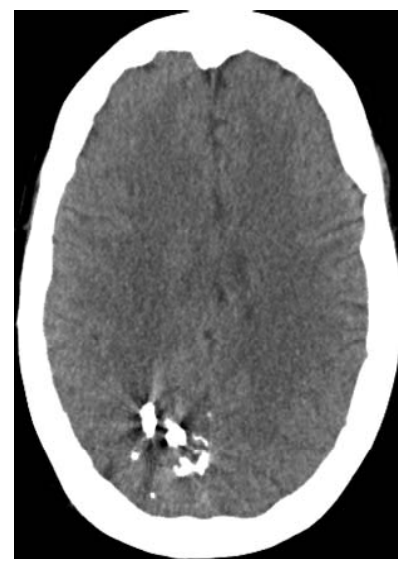

Figure 3. Post-Onyx-embolization CT of the brain demonstrating the Onyx cast.

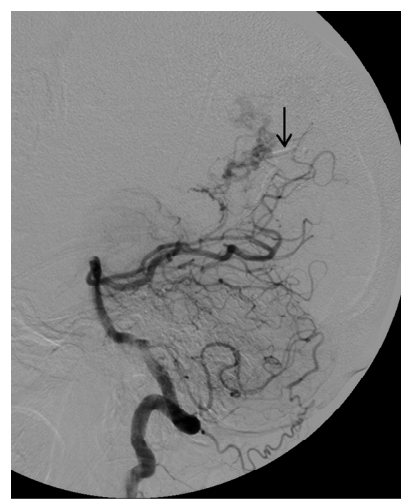

Figure 4. Post-embolization lateral DSA showing Onyx casts within the right PCA feeding vessels (arrows) with reduced flow into the AVM.

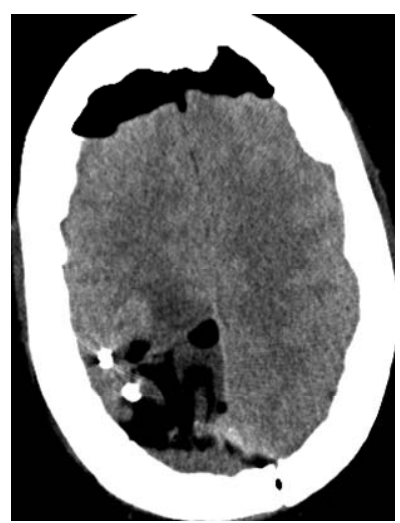

Figure 5. Post-resection non-contrast head CT demonstrating cerebral edema at the resection site as well as pneumocephalus. No hemorrhage is visualized.

However, with her systolic blood pressure 110 - 120 $\mathrm{mmHg}$ in the ensuing hours the patient's left-sided neglect and hemiparesis had returned. Her systolic blood pressure was then reduced again to $90-100 \mathrm{mmHg}$ leading to resolution of her hemiparesis and neglect. After remaining stable over the next few days at systolic pressures less than $100 \mathrm{mmHg}$, her blood pressure parameters were slowly weaned and her neurologic examination remained stable. At the end of her hospital stay, her left-sided hemineglect had improved and her left upper and lower extremities exhibited $4+/ 5$ strength. She was discharged to rehabilitation in stable condition.

On her return follow-up visit, the patient remained neurologically stable without recurrence of her left-sided symptoms. Her post-operative course was complicated by a craniotomy infection requiring wound washout and antibiotic administration. Post-operative DSA and recent follow-up CT angiography demonstrated complete removal of her AVM (Figures 6 and 7). She is now 2 years out from her surgery and remains neurologically intact except for a very mild neglect. Her functional status is excellent.

\section{Discussion}

The present case illustrates hyperperfusion as a causative etiology of neurologic deficit following excision of an AVM and the important role of blood pressure control in ameliorating this phenomenon. Previous studies have demonstrated that staged embolization preceding obliteration can minimize the risk of hyperperfusion injury, while the presence of residual AVM can be a significant contributing factor $[14,17,18]$. However, hyperemic com-
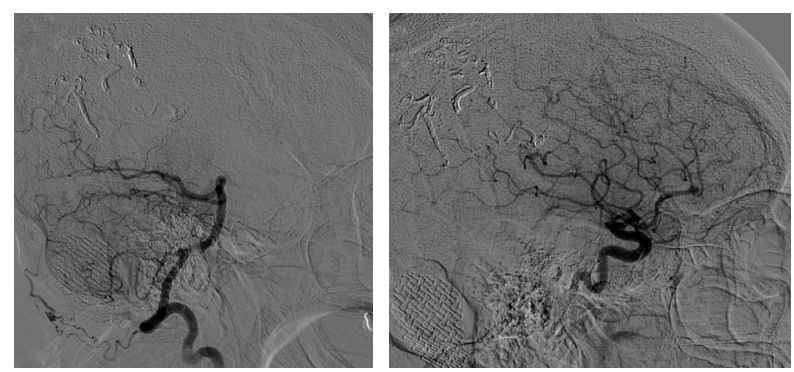

Figure 6. Post-resection, late-arterial phase DSA after right vertebral artery injection (left panel) and right internal carotid artery injection (right panel) demonstrating no further filling of the AVM.

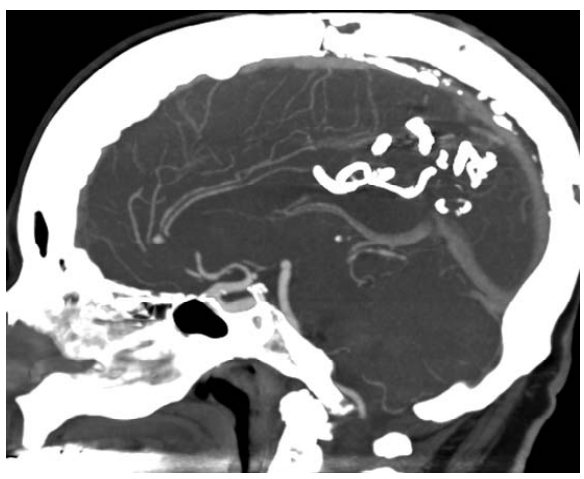

Figure 7. Sagittal CTA of the brain demonstrating Onyx cast and absence of contrast filling of the AVM. 
plications still occurred in this patient after two-stage embolization and complete resection.

There have been several mechanisms proposed to explain hyperemic complications in the setting of complete resection, and the phenomenon of normal perfusion pressure breakthrough has been debated since its introduction in 1978. As previously outlined, the NPPB hypothesis suggests that AVMs may predispose the surrounding vasculature to vasomotor paralysis and potentially lead to cerebral edema or hemorrhage following resection. Support for this theory includes single photon emission computed tomography (SPECT) evidence demonstrating diminished vasoreactivity to acetazolamide in AVM patients and reports of decreased $\mathrm{CO}_{2}$ reactivity in local vasculature during resection $[19,20]$. These studies further indicated that patients with decreased vasoreactivity were at increased risk for developing complications arising from normal perfusion pressure breakthrough.

Despite empirical evidence supporting the existence of autoregulatory dysfunction, there is still controversy attributing hyperemic complications to vasomotor paralysis. Many studies have shown that the majority of postoperative patients demonstrate adequate autoregulatory capacity after AVM resection [10,19,21,22]. Young et al. demonstrated that increasing systemic arterial pressure after complete obliteration did not further elevate cerebral perfusion pressure, even in patients with hyperemic complications [12,21]. Meyer and coworkers then measured cortical oxygen saturation surrounding AVMs before and after resection and reproduced the findings of Young et al., showing once again that diminished autonomic reactivity was not responsible for deterioration in their patient population [23]. Although these reports suggest most patients demonstrate appropriate autoregulation, it is unclear how often NPPB plays a role in hyperemic complications based on these studies. According to Young et al., only 2 patients out of 25 developed cerebral swelling or hemorrhage, and it is unclear if impaired vasoreactivity were even at play in these individuals.

As a result of conflicting evidence surrounding the NPPB hypothesis, other theories have been developed to explain hyperperfusion-induced complications. Occlusive hyperemia is an alternative mechanism potentially explaining cerebral edema and hemorrhage following AVM resection. This theory suggests that venous outflow obstruction and stagnant arterial flow adjacent to the AVM are the primary cause of hyperperfusion injury following complete removal of the nidus $[9,14]$. Several studies have provided evidence consistent with this hypothesis. Al-Rodhan and colleagues, who were the first to propose occlusive hyperemia, reported that $74 \%$ of patients with hyperemic complications following com- plete resection showed impairment of venous drainage on imaging [14]. Schaller et al. later reported that patients with preserved draining veins following ablation did not experience hyperemic complications in their data set, whereas patients developing hyperperfusion injury all had obliterated draining veins [24].

While NPPB and occlusive hyperemia offer different mechanisms to explain hyperemic complications, these models may not be contradictory. Instead, they may be a manifestation of a continuum of vascular compromise within the neurovascular architecture after AVM obliteration [25]. In addition, it has also been reported that perinidal capillaries in both animal and human models lack adequate astrocyte foot processes and basement membranes. This abnormal vascular territory may cause a disruption of the blood-brain barrier that ultimately contributes to edema and hemorrhage after AVM ablation $[15,16]$. Furthermore, residual AVM following excision and accidental lesions of surrounding vessels have also been shown to contribute to hyperemic complications $[12,13]$. In light of these findings, it is possible that a combination of neurovascular and hemodynamic events can predispose patients to hyperemic complications, further fueling the debate over the underlying cause of hyperperfusion-induced injury.

We believe that the clinical course of our patient supports the NPPB theory of hyperperfusion-induced injury. Despite imaging demonstrating there was no residual AVM following resection, the patient developed neurological deficits in the immediate postoperative period. Aggressive systemic hypotension then improved clinical symptoms repeatedly, while a brief period of normotension triggered a return of neurological deficits. As a result, there was a direct correlation between improvement and degradation of neurological status in relation to arterial pressure. This case suggests that the intrinsic autoregulatory capacity was altered in our patient, and that aggressive hypotension was necessary to compensate for diminished autonomic reactivity.

Hyperperfusion injury has been reported as a complicating factor after other vascular interventions as well. Following carotid endarterectomy, cerebral blood flow can increase above basal levels to produce the same hyperemic complications seen after AVM removal [26]. In the setting of hyperperfusion following both AVM resection and carotid endarterectomy, several imaging modalities, such as perfusion computed tomography (CT), have been used to evaluate cerebral blood flow. Perfusion CT is an effective imaging technique that provides rapid information on hemodynamic alterations. By comparing regional cerebral blood flow (rCBF), mean transit time (MTT), and time to peak (TTP), areas of altered perfusion can be identified [27,28].

Using CT perfusion imaging, Chang et al. demon- 
strated that hyperperfusion injury could be accurately identified after carotid endarterectomy by observing differences in rCBF and TTP [29]. Recently, Vasquez et al. used CT perfusion to show decreased MTT in a patient with cerebral hyperperfusion syndrome, which resolved with the patient's symptoms following blood pressure reduction [28]. One limitation of our report is that CT perfusion was not obtained during the evolution of the patient's neurological deficits because a reduction in blood pressure repeatedly resulted in symptomatic improvement.

Over the last 40 years, studies have suggested various techniques to reduce the incidence of edema and hemorrhage after AVM obliteration [18,30,31]. Staged embolization of large AVMs, proximal arterial feeder ligation, and systemic hypotension have all been proposed to limit hyperperfusion injury $[13,17,18]$. Generally, these protocols attempt to lower the potentially harmful elevated pressure in arterial feeders following partial nidus resection [13]. However, this case is unique because the patient had neurologic deficits clearly related to blood pressure immediately following resection of the AVM without evidence of residual AVM or hemorrhage. In addition, it provides a direct temporal relationship that illustrates the cause and effect association between hyperperfusion and induced systemic hypotension with resolution of neurological symptoms. This case adds to the growing literature that concludes strict blood pressure control can be useful in the setting of complete AVM obliteration [7,13,32].

\section{Conclusion}

Hyperperfusion complications after AVM resection are poorly understood. We present a patient that developed cerebral edema and neurological deficits after AVM resection that responded repeatedly to aggressive systemic hypotension. This case provides further evidence that normal perfusion pressure breakthrough plays a role in hyperperfusion-induced injury following AVM excision, and that blood pressure control can be vital in managing hyperemic complications following complete resection of cerebral AVMs.

\section{REFERENCES}

[1] W. A. Friedman, D. L. Blatt, F. J. Bova, J. M. Buatti, W. M. Mendenhall and P. S. Kubilis, "The Risk of Hemorrhage after Radiosurgery for Arteriovenous Malformations," Journal of Neurosurgery, Vol. 84, No. 6, 1996, pp. 912-919. http://dx.doi.org/10.3171/jns.1996.84.6.0912

[2] D. Kondziolka, J. C. Flickinger and L. Dade Lunsford, "Clinical Research in Stereotactic Radiosurgery: Lessons Learned from over 10,000 Cases,” Neurological Research, Vol. 33, No. 8, 2011, pp. 792-802.

http://dx.doi.org/10.1179/1743132811Y.0000000034
[3] S. L. Ondra, H. Troupp, E. D. George and K. Schwab, "The Natural History of Symptomatic Arteriovenous Malformations of the Brain: A 24-Year Follow-Up Assessment," Journal of Neurosurgery, Vol. 73, No. 3, 1990, pp. 387-391. http://dx.doi.org/10.3171/jns.1990.73.3.0387

[4] D. J. Englot, W. L. Young, S. J. Han, C. E. McCulloch, E. F. Chang and M. T. Lawton, "Seizure Predictors and Control after Microsurgical Resection of Supratentorial Arteriovenous Malformations in 440 Patients," Neurosurgery, Vol. 71, No. 3, 2012, pp. 572-580. http://dx.doi.org/10.1227/NEU.0b013e31825ea3ba

[5] R. F. Spetzler and N. A. Martin, "A Proposed Grading System for Arteriovenous Malformations,” Journal of Neurosurgery, Vol. 65, No. 4, 1986, pp. 476-483. http://dx.doi.org/10.3171/jns.1986.65.4.0476

[6] M. G. Hamilton and R. F. Spetzler, "The Prospective Application of a Grading System for Arteriovenous Malformations," Neurosurgery, Vol. 34, No. 1, 1994, pp. 2-6. http://dx.doi.org/10.1227/00006123-199401000-00002

[7] M. K. Morgan, M. Winder, N. S. Little, S. Finfer and E. Ritson, "Delayed Hemorrhage Following Resection of an Arteriovenous Malformation in the Brain," Journal of Neurosurgery, Vol. 99, No. 6, 2003, pp. 967-971. http://dx.doi.org/10.3171/jns.2003.99.6.0967

[8] R. F. Spetzler, C. B. Wilson, P. Weinstein, M. Mehdorn, J. Townsend and D. Telles, "Normal Perfusion Pressure Breakthrough Theory,” Clinical Neurosurgery, Vol. 25, 1978, pp. 651-672.

[9] N. R. Al-Rodhan, "Occlusive Hyperemia Remains the Most Logical Explanation for the Hemodynamic Complications of Resected Intracerebral Arteriovenous Malformations," Journal of Neurosurgical Anesthesiology, Vol. 7, No. 3, 1995, pp. 208-210. http://dx.doi.org/10.1097/00008506-199507000-00019

[10] M. D. Alexander, E. S. Connolly and P. M. Meyers, "Revisiting Normal Perfusion Pressure Breakthrough in Light of Hemorrhage-Induced Vasospasm," World Journal of Radiology, Vol. 2, No. 6, 2010, pp. 230-232. http://dx.doi.org/10.4329/wjr.v2.i6.230

[11] W. L. Young, I. Prohovnik, E. Ornstein, N. Ostapkovich, M. B. Sisti, R. A. Solomon and B. M. Stein, "The Effect of Arteriovenous Malformation Resection on Cerebrovascular Reactivity to Carbon Dioxide,” Neurosurgery, Vol. 27, No. 2, 1990, pp. 257-266. http://dx.doi.org/10.1227/00006123-199008000-00015

[12] W. L. Young, A. Kader, I. Prohovnik, E. Ornstein, L. H. Fleischer, N. Ostapkovich, L. D. Jackson and B. M. Stein, "Pressure Autoregulation Is Intact after Arteriovenous Malformation Resection," Neurosurgery, Vol. 32, No. 4, 1993, pp. 491-496. http://dx.doi.org/10.1227/00006123-199304000-00001

[13] T. F. Massoud, G. J. Hademenos, W. L. Young, E. Gao and J. Pile-Spellman, "Can Induction of Systemic Hypotension Help Prevent Nidus Rupture Complicating Arteriovenous Malformation Embolization? Analysis of Underlying Mechanism Achieved Using a Theoretical Model,” American Journal of Neuroradiology, Vol. 21, No. 7, 2000, pp. 1255-1267.

[14] N. R. Al-Rodhan, T. M. Sundt Jr., D. G. Piepgras, D. A. 
Nichols, D. Rufenacht and L. N. Stevens, "Occlusive Hyperemia: A Theory for the Hemodynamic Complications Following Resection of Intracerebral Arteriovenous Malformations," Journal of Neurosurgery, Vol. 78, No. 2, 1993, pp. 167-175. http://dx.doi.org/10.3171/jns.1993.78.2.0167

[15] L. H. Sekhon, M. K. Morgan and I. Spence, "Normal Perfusion Pressure Breakthrough: The Role of Capillaries,” Journal of Neurosurgery, Vol. 86, No. 3, 1997, pp. 519-524. http://dx.doi.org/10.3171/jns.1997.86.3.0519

[16] J. Tu, M. A. Stoodley, M. K. Morgan and K. P. Storer, "Ultrastructure of Perinidal Capillaries in Cerebral Arteriovenous Malformations,” Neurosurgery, Vol. 58, No. 5, 2006, pp. 961-970. http://dx.doi.org/10.1227/01.NEU.0000210248.39504.B5

[17] J. Z. Zhao, S. Wang, J. S. Li, D. L. Sui, Y. L. Zhao and Y. Zhang, "Combination of Intraoperative Embolisation with Surgical Resection for Treatment of Giant Cerebral Arteriovenous Malformations," Journal of Clinical Neuroscience, Vol. 7, Suppl. 1, 2000, pp. 54-59. http://dx.doi.org/10.1054/jocn.2000.0713

[18] H. Kinouchi, K. Mizoi, A. Takahashi, M. Ezura and T. Yoshimoto, "Combined Embolization and Microsurgery for Cerebral Arteriovenous Malformation,” Neurologia Medico-Chirurgica (Tokyo), Vol. 42, No. 9, 2002, pp. 372-378. http://dx.doi.org/10.2176/nmc.42.372

[19] K. Ogasawara, K. Yoshida, Y. Otawara, M. Kobayashi, S. Yasuda, M. Doi and A. Ogawa, "Cerebral Blood Flow Imaging in Arteriovenous Malformation Complicated by Normal Perfusion Pressure Breakthrough,” Surgical Neurology, Vol. 56, No. 6, 2001, pp. 380-384. http://dx.doi.org/10.1016/S0090-3019(01)00655-3

[20] G. H. Barnett, J. R. Little, Z. Y. Ebrahim, S. C. Jones and H. T. Friel, "Cerebral Circulation during Arteriovenous Malformation Operation,” Neurosurgery, Vol. 20, No. 6, 1987, pp. 836-842. http://dx.doi.org/10.1227/00006123-198706000-00003

[21] W. L. Young, A. Kader, E. Ornstein, K. Z. Baker, N. Ostapkovich, J. Pile-Spellman, P. Fogarty-Mack and B. M. Stein, "Cerebral Hyperemia after Arteriovenous Malformation Resection Is Related to 'Breakthrough' Complications but Not to Feeding Artery Pressure. The Columbia University Arteriovenous Malformation Study Project,” Neurosurgery, Vol. 38, No. 6, 1996, pp. 1085-1093. http://dx.doi.org/10.1227/00006123-199606000-00005

[22] A. Kader and W. L. Young, "The Effects of Intracranial Arteriovenous Malformations on Cerebral Hemodynamics," Neurosurgery Clinics of North America, Vol. 7, No. 4, 1996, pp. 767-781.

[23] B. Meyer, C. Schaller, C. Frenkel, B. Ebeling and J. Schramm, "Distributions of Local Oxygen Saturation and
Its Response to Changes of Mean Arterial Blood Pressure in the Cerebral Cortex Adjacent to Arteriovenous Malformations,” Stroke, Vol. 30, No. 12, 1999, pp. 2623-2630. http://dx.doi.org/10.1161/01.STR.30.12.2623

[24] C. Schaller, H. Urbach, J. Schramm and B. Meyer, "Role of Venous Drainage in Cerebral Arteriovenous Malformation Surgery, as Related to the Development of Postoperative Hyperperfusion Injury,” Neurosurgery, Vol. 51, No. 4, 2002, pp. 921-927.

[25] B. E. Zacharia, S. Bruce, G. Appelboom and E. S. Connolly Jr., “Occlusive Hyperemia versus Normal Perfusion Pressure Breakthrough after Treatment of Cranial Arteriovenous Malformations," Neurosurgery Clinics of North America, Vol. 23, No. 1, 2012, pp. 147-151. http://dx.doi.org/10.1016/j.nec.2011.09.005

[26] W. N. van Mook, R. J. Rennenberg, G. W. Schurink, R. J. van Oostenbrugge, W. H. Mess, P. A. Hofman and P. W. de Leeuw, "Cerebral Hyperperfusion Syndrome," Lancet Neurology, Vol. 4, No. 12, 2005, pp. 877-888. http://dx.doi.org/10.1016/S1474-4422(05)70251-9

[27] N. Khandelwal, “CT Perfusion in Acute Stroke,” Indian Journal of Radiology and Imaging, Vol. 18, No. 4, 2008, pp. 281-286. http://dx.doi.org/10.4103/0971-3026.43837

[28] R. A. Vasquez, M. F. Waters, C. J. Skowlund, J. Mocco and B. L. Hoh, "Computed Tomographic Perfusion Imaging of Non-Hemorrhagic Cerebral Hyperperfusion Syndrome and Reversal Following Medical Treatment after Carotid Artery Angioplasty and Stenting," Journal of Neurointerventional Surgery, Vol. 4, No. 3, 2012, p. e2. http://dx.doi.org/10.1136/jnis.2010.003558

[29] C. H. Chang, T. Y. Chang, Y. J. Chang, K. L. Huang, S. C. Chin, S. J. Ryu, T. C. Yang and T. H. Lee, "The Role of Perfusion Computed Tomography in the Prediction of Cerebral Hyperperfusion Syndrome,” PLoS One, Vol. 6, No. 5, 2011, p. e19886. http://dx.doi.org/10.1371/journal.pone.0019886

[30] C. Lundqvist, G. Wikholm and P. Svendsen, "Embolization of Cerebral Arteriovenous Malformations: Part IIAspects of Complications and Late Outcome," Neurosurgery, Vol. 39, No. 3, 1996, pp. 460-467.

[31] M. K. Morgan, L. H. Sekhon, S. Finfer and V. Grinnell, "Delayed Neurological Deterioration Following Resection of Arteriovenous Malformations of the Brain,” Journal of Neurosurgery, Vol. 90, No. 4, 1999, pp. 695-701. http://dx.doi.org/10.3171/jns.1999.90.4.0695

[32] I. Nakagawa, S. Kawaguchi, J. Iida, H. Hirabayashi and T. Sakaki, "Postoperative Hyperperfusion Associated with Steal Phenomenon Caused by a Small Arteriovenous Malformation,” Neurologia Medico-Chirurgica (Tokyo), Vol. 45, No. 7, 2005, pp. 363-366. http://dx.doi.org/10.2176/nmc.45.363 